\title{
Ärztliche Fehler: Häufung bei Depression, aber nicht bei Burn-out
}

Chefredakteur Dr. med. Dirk Einecke

\section{STRIKTE BLUTZUCKERKONTROLLE SCHÄDLICH?}

\section{Immer mehr Zweifel}

Letzte Woche berichteten wir an dieser Stelle über die vorzeitig abgebrochene ACCORD-Studie, die bei intensiver Diabetes-Therapie $\left(\mathrm{HbA}_{1 \mathrm{c}} 6,4 \%\right)$ nach vier Jahren eine etwas höhere Sterblichkeit beobachtete als unter weniger intensiver Behandlung $\left(\mathrm{HbA}_{1 \mathrm{c}} 7,5 \%\right)$. Diese Ergebnisse sind schwer zu verstehen. Denn die zeitgleich publizierte STENO-2-Studie zeigt das Gegenteil.

\section{SCHLECHTER SEX}

\section{Empfehlen Sie Pumps!}

Bei Frauen mit sexuellen Problemen kann eine einfache Maßnahme Abhilfe schaffen: Das Tragen von Schuhen mit hohen Absätzen. Die scharfen Treter sollen dabei ihr therapeutisches Potenzial weder über die Optik (Mann schaut wieder hin) noch über die Psychologie (Frau fühlt sich besser) entfalten. Der Grund sei vielmehr ein anatomischer: Das Balancieren auf hohen Schuhen stärkt die Beckenmuskulatur, berichtet die Süddeutsche Zeitung unter Be-

\section{RÜCKENPROBLEME}

\section{Teure Therapien lösen sie nicht}

Muskuloskelettale Probleme im Rückenund Nackenbereich sind in Deutschland die häufigste Ursache für AU-Tage. Wie Autoren in JAMA schreiben, sind auch in den USA die Behandlungskosten für diese Erkrankungen stark angestiegen. Von 1997 bis 2005 haben sich die Ausgaben für Rückenpatienten bei ambulanten

\section{RISIKOFAKTOR ÜBERGEWICHT}

\section{Dicke haben öfter Krebs}

Übergewicht erhöht nicht nur das Risiko für ein metabolisches Syndrom und Diabetes, sondern auch für einige Krebsarten. Bei Männern geht ein Anstieg des BMI um $5 \mathrm{~kg} / \mathrm{m}^{2}$ einher mit einem erhöhten Risiko für Adenokarzinome des Ösophagus (RR 1,52), Schilddrüsen- (RR 1,33) und Nierenkarzinome (RR 1,24). Bei Frauen steigt das Risiko für Endometrium- (RR $1,59)$, Gallenblasen- (RR 1,59) und Nieren-
Nun melden sich die Autoren einer weiteren Studie (ADVANCE), die ebenfalls den Nutzen einer intensiven Diabetestherapie untersucht. Auch diese Studie gibt in einer Interimsanalyse keine Hinweise auf eine erhöhte Sterblichkeit bei strammer BZ-Einstellung. Die ADVANCE-Autoren betonen, dass sie mehr als doppelt so viele Patienten untersuchten wie die ACCORDStudie. Die BZ-Werte waren identisch.

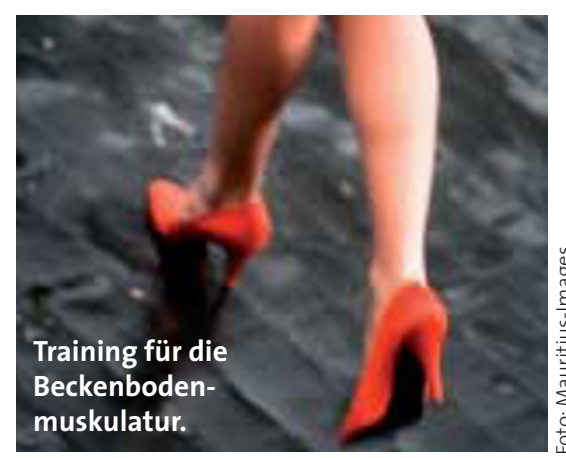

rufung auf eine urologische Studie aus Italien.

Patienten um 36\% erhöht. Am Ergebnis (gemessen an funktioneller Behinderung und Arbeitsausfällen) hat sich jedoch kaum etwas geändert. Die Beschwerden der Patienten wurden 2005 sogar als schwerwiegender geschildert als zehn Jahre zuvor.

JAMA 2008;299:656-64

\section{BLUTZUCKER WIRD ERHÖHT}

\section{Sollen Diabetiker auf Kaffee verzichten?}

Regelmäßiger Konsum von koffeinhaltigen Getränken kann die Blutzuckereinstellung von Typ-2-Diabetikern verschlechtern. Das zeigt eine Studie mit zehn Typ-2-Diabetikern, deren Blutglukosespiegel kontinuierlich überwacht wurden. An Tagen, an denen sie eine vier Tassen Kaffee entsprechende Koffeinmenge erhielten, waren ihre Blutzuckerwerte durchschnittlich um $8 \%$ höher als an Tagen, an denen sie ein Placebo einnahmen. Auch der postprandiale BZ-Anstieg fiel an Koffeintagen deutlich höher aus. Diabetes Care. 2008;31:221-22

\section{ALTE, ROBUSTE ZAHNPLOMBEN}

\section{Amalgam macht nicht krank}

Amalgam enthält Quecksilber. Daher steht es unter Verdacht, systemische $\mathrm{Ne}$ benwirkungen, vor allem neurologischer oder psychiatrischer Natur, auszulösen. Zu Unrecht, wie eine Studie der EU ergeben hat. Danach stellen Amalgamfüllungen kein Gesundheitsrisiko dar. Lediglich harmlose lokale Reaktionen könnten in seltenen Fällen auftreten. Die Quecksilberexposition ist übrigens am höchsten, wenn das Amalgam eingesetzt oder herausgenommen wird. Es gibt daher keine medizinische Begründung, intakte Amalgamfüllungen zu ersetzen.

\section{ARZT MIT DEPRESSION}

\section{Das Risiko für Behandlungsfehler steigt}

Unter den Arbeitsbedingungen im Gesundheitswesen leiden nicht nur die Ärzte, sondern auch die ärztliche Kunst. So wurde an drei Kinderkliniken in den USA bei $20 \%$ der jungen Ärzte eine Depression und bei $74 \%$ ein Burn-out diagnostiziert. Während die ausgebrannten Ärzte genauso gut arbeiteten wie ihre Kollegen ohne Burn-out, wurde durch eine Depression die Fehlerrate deutlich erhöht. Den betroffenen Ärzten unterliefen rund sechsmal so viele Behandlungsfehler wie Ärzten ohne Depression.

Brit Med J, online 15. Februar 2008 